\title{
Lack of protease inhibitor resistance following treatment failure - too good to be true?
}

\author{
John A. Bartlett, MD
}

Department of Medicine and the Duke Global Health Institute, Duke University Medical Center, Durham, North Carolina, USA.

\begin{abstract}
A 29-year-old man with recently diagnosed HIV infection and a CD4 cell count of $225 / \mathrm{mm}^{3}$ began treatment with atazanavir $(300 \mathrm{mg})$, ritonavir (100 mg), emtricitabine (200 mg), and tenofovir (300 mg) daily. For 18 months, he was treatment adherent and his plasma HIV RNA level was below the limit of detection. He then began a relationship with a new partner, who introduced him to methamphetamines. His medication adherence became erratic, and he missed appointments in clinic. Eventually. he was hospitalized for rehabilitation, and he resumed taking his medications on schedule. Following his discharge, he was found to have a plasma HIV RNA level of 11,400 copies/ml. Genotypic resistance testing revealed only an $\mathrm{M184V}$ mutation associated with emtricitabine resistance. A decision regarding his next treatment regimen needs to be made.
\end{abstract}

\section{Current therapy}

The clinical dilemma posed by this patient is commonly encountered by clinicians treating HIV-infected patients. This patient has clearly failed his treatment regimen, but genotypic resistance tests have failed to provide much guidance in choosing the next regimen.

Protease inhibitors (PI) represent valuable tools in the armamentarium of clinicians treating HIV-infected persons. Clinical trials in both treatment-naive and treatment-experienced subjects have demonstrated outstanding PI activity, and regimens containing PI may be recommended for use across these different patient populations (1). Indeed, two of the four recommended regimens for the initial treatment of HIV infections include PI (atazanavir and darunavir) (1).

\section{Knowledge gap}

Challenges for the PI class have included the need for pharmacologic boosting with some members of the class, especially if patients are more heavily treatment experienced and most notably if they are PI experienced (1). Numerous studies of patients who fail PI have not identified mutations in the protease gene associated with resistance (2-5). In a systematic review of failure of first-line antiretroviral therapy, per-

Conflict of interest: The author has declared that no conflict of interest exists.

Citation for this article: J Clin Invest. 2013; 123(9):3704-3705. doi:10.1172/JCI71784. sons failing PI-containing regimens had the least accumulation of drug resistance mutations, both within the protease gene and, to a lesser extent, in the reverse transcriptase gene (6). This conundrum has baffled investigators for many years and defies the evolutionary logic that virologic failure should be accompanied by mutations in the drug target. From a clinical perspective, the apparent lack of resistance complicates the choice of a PI in future combination regimens.

\section{Research advances}

The observations of Rabi et al. shed new light on the inhibition of the HIV life cycle and alternative mechanisms of resistance to PIs (7). First, the authors have utilized a series of clever experiments to identify the inhibitory effect of PIs on several steps in the life cycle, including virus entry, reverse transcription, and posttranscription events. Previous descriptions of PI activity have identified inhibition of the proteolytic cleavage of precursor proteins, a relatively late step in the HIV life cycle, as the mechanism for their antiretroviral effect. Second, in nine subjects who failed PI-containing regimens with no detectable mutations in their protease genes in genotypic assays, they have identified env mutations with additional sequencing. Given that commercially available genotypic resistance assays do not sequence and report env genes, clinicians may be falsely assured that PI resistance has not occurred.

Certainly these observations are intriguing and may prove to be very important in clinical management. However, it remains uncertain how commonly the env mutations occur in patients failing PIs. Additional studies are needed to address this question and may eventually lead to a need for expanded resistance assays, including env sequencing, to optimize selection of subsequent PIs.

Rabi et al. speculate on three resistance mechanisms to explain PI failure in the absence of protease mutations. The first involves nonadherence by the patient, and due to the absence of the drug, no resistance mutations in the protease gene are selected. The second explanation involves the unique pharmacologic and pharmacodynamic properties of PI with high potency and relatively short half-lives, resulting in brief periods within the mutant selection window. Finally, the newly described env mutations may compromise PI activity. A fourth mechanism involving mutations at proteolytic cleavage sites has also been associated with PI resistance in a limited number of patients (8).

\section{Recommendations}

What are the implications for HIV clinical care? Until larger studies are completed assessing the frequency of env mutations, clinicians are likely to follow a simple paradigm. On the detection of virologic failure, reinforcing adherence to medications will result in resuppression of some patients. If resuppression does not occur, conventional genotypic resistance testing may identify mutations in the protease gene and guide the choice of subsequent PIs. However, in patients with a wild-type protease gene, clinicians will remain uncertain about the optimal choice of a PI for the next regimen. They will make an educated guess on the PI choice and will need to closely follow their patients on the new treatment regimen.

\section{Acknowledgments}

The author receives salary support from NIH awards P30AI64518, U01AI067854, D43CA153722, and D43TW06732.

Address correspondence to: John A. Bartlett, Department of Medicine and the 
Duke Global Health Institute, Box 3238, Duke University Medical Center, Durham, North Carolina 27710, USA. Phone: 919.681.8043; Fax: 919.681.7748; E-mail: jab5@duke.edu.

1. Panel on Antiretroviral Guidelines for Adults and Adolescents. Guidelines for the use of antiretroviral agents in the treatment of HIV-1-infected adults and adolescents. Department of Health and Human Services Web site. http://aidsinfo.nih. gov/ContentFiles/AdultandAdolescentGL.pdf. Accessed June 27, 2013.
2. Kempf D, et al. Incidence of resistance in a doubleblind study comparing lopinavir/ritonavir plus stavudine and lamivudine to nelfinavir plus stavudine and lamivudine. J Inf Dis. 2004;189(1):51-60.

3 . Eron J, et al. The KLEAN study of fosamprenavir-ritonavir versus lopinavir-ritonavir, each in combination with abacavir-lamivudine, for initial treatment of HIV infection over 48 weeks: a randomized non-inferiority trial. Lancet. 2006; 368(9534):476-482.

4. Riddler $\mathrm{S}$, et al. Class-sparing regimens for initial treatment of HIV-1 infection. N Engl J Med. 2008; 358(20):2095-2106

5. Daar E, et al. Atazanavir plus ritonavir or efavirenz as part of a 3-drug regimen for initial treatment of HIV-1. Ann Intern Med. 2011;154(7):445-456.

6 . Bartlett J, et al. Minimizing resistance consequences after virologic failure on initial combination therapy. J Acquir Immune Defic Syndr. 2006; 41(3):323-331.

7. Rabi SA, et al. Multi-step inhibition explains HIV-1 protease inhibitor pharmacodynamics and resistance. J Clin Invest. 2013;123(9):3848-3860.

8. Côté HC, Brumme ZL, Harrigan PR. Human immunodeficiency virus type 1 protease cleavage site mutations associated with protease inhibitor cross-resistance selected by indinavir, ritonavir, and/or saquinavir. J Virol. 2001;75(2):589-594. 